\title{
A Time-Varied Probabilistic ON/OFF Switching Algorithm for Cellular Networks
}

\author{
Nadhir Ben Rached, Student Member, IEEE, Hakim Ghazzai, Member, IEEE, Abdullah Kadri, Senior \\ Member, IEEE, and Mohamed-Slim Alouini, Fellow, IEEE
}

\begin{abstract}
In this letter, we develop a time-varied probabilistic on/off switching planning method for cellular networks to reduce their energy consumption. It consists in a risk-aware optimization approach that takes into consideration the randomness of the user profile associated with each base station (BS). The proposed approach jointly determines (i) the instants of time at which the current active BS configuration must be updated due to an increase or decrease of the network traffic load, and (ii) the set of minimum BSs to be activated to serve the networks' subscribers. Probabilistic metrics modeling the traffic profile variation are developed to trigger this dynamic on/off switching operation. Selected simulation results are then performed to validate the proposed algorithm for different system parameters.
\end{abstract}

Index Terms-Base station on/off switching, cellular networks, risk level, time-varied probabilistic approach.

\section{INTRODUCTION}

The number of mobile users has grown rapidly over the last decades. They are increasing at a rate of approximately 1.5 to 2 times a year. Therefore, more base stations (BSs) are being installed to improve the quality of service (QoS) of the network and increase its capacity to deal with this massive traffic increase. This leads to an enormous energy consumption in cellular networks that may cause negative environmental and economical impacts. Therefore, there is a pressing need to employ new technologies to reduce the exponential growth of energy consumption [1].

$\mathrm{BS}$ on/off switching is a promising technique that has shown a great potential in contributing to the reduction of energy consumption of cellular networks [2]. In its simpler form, the idea behind BS on/off switching is to turn off lightly loaded BSs while maintaining a reliable QoS by serving the corresponding users via neighbor BSs. Several studies have been carried out to reduce energy consumption via the employment of BS on/off switching strategies. For example, in [3], a traffic load balancing has been proposed to achieve tradeoffs between two network utilities; namely the average traffic delivery latency and the green energy utilization. The authors in [4] have investigated the impact of optimizing the BS statuses on the energy-delay tradeoff. A comprehensive survey about BS on/off switching strategies is provided in [5].

BS on/off methods existing in the literature propose either instantaneous on/off switching based on real-time and instantaneous network statistics or pre-planned on/off switching approaches based on average statistics of the networks. The former approaches are usually unpractical for realistic

Nadhir Ben Rached and Mohamed-Slim Alouini are with King Abdullah University of Science and Technology (KAUST), Thuwal, Makkah Province, Saudi Arabia. E-mails: \{nadhir.benrached, slim.alouini\}@kaust.edu.sa.

Hakim Ghazzai and Abdullah Kadri are with Qatar Mobility Innovations Center (QMIC), Qatar University, Doha, Qatar. E-mails: \{hakimg, abdullahk\}@qmic.com. scenarios due to high $\mathrm{BS}$ on/off oscillation rates and they can be applied for micro-sleep modes only where BSs cannot be completely turned off [6]. The oscillation problem has been considered in [7] using hysteresis switch policy. The latter methods are often applied for periodic/fixed time intervals where the network statistics are averaged over the time and the space (i.e., spatial and temporal distributions per cell). The above explanation differentiates our work from the existing on/off switching methods. In fact, we incorporate, via a risk aware manner measured by a risk level set by the operator, a time varied probabilistic on/off switching approach when optimizing the BSs' operation modes. More specifically, the proposed algorithm is divided into two steps. First, we determine through a risk-aware probabilistic manner the time period during which the current configuration could be kept while satisfying a certain QoS level and BS power constraints. This step strongly depends on the traffic profile of each BS aiming at adaptively updating their statuses depending on the uncertain future behavior of the traffic. This constitutes the first major difference with previous works wherein the BSs' states are updated each fixed time period. The second step of our algorithm determines the BS configuration during each time interval identified by the first step. The second step is also performed with a probabilistic approach wherein the BSs are selected such that the average total power consumption of the network is minimized while ensuring, with high probability, a safe network operation.

\section{SySTEM MODEL}

We consider a cellular network composed of $N$ BSs. The power consumption at time $t$ of BS $n$, where $n \in\{1, \ldots, N\}$, denoted by $P_{n}\left(t, N_{n}(t)\right)$, can be expressed as follows [6]:

$$
P_{n}\left(t, N_{n}(t)\right)=a P_{u} N_{n}(t)+b,
$$

where $a$ is a factor that scales with the radiated power due to amplifier and feeder losses, $b$ models an offset of site power which is consumed independently of the average transmit power, and $P_{u}$ is the consumed power per unit of traffic that we assume to be fixed and uniform. $N_{n}(t)$ denotes the traffic profile corresponding to the number of users of the $n^{\text {th }}$ BS and at time $t$. It is assumed to be random and modeled as:

$$
N_{n}(t)=\bar{N}_{n}(t)+\delta_{n}(t),
$$

where $\bar{N}_{n}(t)$ is the average number of users of BS $n$ at time $t$ and $\delta_{n}(t)$ is a random variable measuring the error between the exact number of users and its expected value. Note that our proposed approach is not restricted to the power model given in (1). Any other power model can be adopted in this framework without affecting the applicability of our proposed approach. For instance, we can consider a nonlinear power 
model or we adopt a dynamic power allocation in which $P_{u}$ depends on the number of users connected to each BS.

We define the vector $\epsilon$ whose $n^{\text {th }}$ entry represents the status of BS $n$ as follows

$$
\epsilon=\left\{\begin{array}{l}
1, \text { if BS } n \text { is switched on, } \\
0, \text { if BS } n \text { is switched off. }
\end{array}\right.
$$

This vector represents the principal decision variable which will be determined for different time periods according to the dynamic statistics of the network.

\section{PRobabilistic BS Configuration Update}

The main objective of this study is to develop, depending on the random users' profile, a time-varied risk-aware probabilistic on/off switching algorithm. The proposed algorithm encompasses two steps. At time $t_{0}$ and for a given BS configuration, the first step aims to identify the maximum time period $\Delta t$ (in a sense that will be discussed later) during which the considered BS configuration could be maintained. Then, at time $t_{0}+\Delta t$, the second step looks for a new best BS configuration that minimizes the power consumption while ensuring a reliable network operation. In other words, it aims to identify the BSs that must be activated and the ones that have to be turned to the sleep mode. This two-step process is repeated continuously during the network operation ${ }^{1}$. This will lead to a reduced on/off switching oscillation rates over not necessarily equal time periods.

\section{A. First Step: Time to Update}

In order to determine the maximum time period during which the current BS configuration $\epsilon$ can be maintained, certain criteria related to the network operation are introduced. They will serve as indicators of whether the considered configuration could be kept in the future or not. The objective is to trigger alerts if the QoS is expected to be violated, the active BSs' capacities will be exceeded (e.g., power budgets), or significant variations of the traffic profile are observed. Assume that, at time $t_{0}$, we have determined the entries of $\boldsymbol{\epsilon}\left(\boldsymbol{\epsilon}_{n}, n \in\{1,2, \cdots, N\}\right)$. Then, at time $t_{0}+\Delta t$, the entries of $\epsilon$ must be updated if the following equation occurs:

$$
p\left(A_{1} \cup A_{2} \cup A_{3}\right) \geq \eta,
$$

where $p(A)$ denotes the probability of an event and the events $A_{i}, i=1,2,3$, are defined as:

- The event $A_{1}$ aims to guarantee that the network QoS is maintained during $\Delta t$. It indicates that a network update is needed if the percentage of served users is less than a certain tolerated level $\mathrm{QoS}_{t h}$ set by the operator ${ }^{2} . A_{1}$ is defined as:

$$
A_{1}=\left\{\frac{\sum_{n=1}^{N} \epsilon_{n} \tilde{N}_{n}\left(t_{0}+\Delta t\right)}{\sum_{n=1}^{N} N_{n}\left(t_{0}+\Delta t\right)} \leq \mathrm{QoS}_{t h}\right\},
$$

where $\tilde{N}_{n}(\cdot)$ is the total number of users served by the $n^{\text {th }}$ BS and is given by:

$$
\tilde{N}_{n}(t)=\left\{\begin{array}{l}
N_{n}(t)+\sum_{\substack{k=1 \\
k \neq n}}^{N} x_{n k} N_{k}(t), \text { if } \boldsymbol{\epsilon}_{n}=1 \\
0, \text { Otherwise. }
\end{array}\right.
$$

\footnotetext{
${ }^{1}$ Unlike previous techniques, the proposed solution is applied for unlimited time horizon and does not necessary require cyclic network operation

${ }^{2}$ Note that other QoS metrics can be considered in this problem. In this paper, we choose to evaluate the QoS based on the traffic load and the number of users successfully served
}

More precisely, $\tilde{N}_{n}(t)$ represents the sum of number of users covered by $\mathrm{BS} n$ in addition to parts of users belonging to other inactive BSs $k$ (i.e., $\boldsymbol{\epsilon}_{k}=0$ ) but offloaded to BS $n$ evaluated by the fraction $x_{n k}, 0 \leq x_{n k} \leq 1$. Note also that, in the case where the offloaded users can be served by multiple $\mathrm{BSs}$ at the same time, the users are associated to the closest BSs.

- The event $A_{2}$ aims to evaluate the variation of the total power consumption of the network between the instants $t_{0}$ and $t=t_{0}+\Delta t$. When the probability of this event is high, the operator may have the possibility to switch off some of its BSs to reduce its total power consumption. $A_{2}$ is defined as:

$A_{2}=\left\{\sum_{n=1}^{N} \epsilon_{n}\left|P_{n}\left(t_{0}, \tilde{N}_{n}\left(t_{0}\right)\right)-P_{n}\left(t_{0}+\Delta t, \tilde{N}_{n}\left(t_{0}+\Delta t\right)\right)\right| \geq \bar{P}\right\}$,

where $\bar{P}$ is a constant representing a power threshold to be pre-defined by the operator.

- The event $A_{3}$ aims to ensure that the power consumption levels of the active BSs are not exceeding the power budget constraint during the period $\Delta t$ and is defined as follows:

$$
A_{3}=\cup_{n=1}^{N}\left\{\epsilon_{n} P_{n}\left(t_{0}+\Delta t, \tilde{N}_{n}\left(t_{0}+\Delta t\right)\right)>\tilde{P}\right\},
$$

where $\tilde{P}$ is the BS power budget. When it occurs with high probability, this event indicates that at least one of the BSs is violating its capacity. In this case, the operator would need to update its BS configuration. In a nutshell, the above three indicators are measures of the network variation and serve, when no alert is detected, to ensure a safe network operation.

In (3), $\eta$ is a risk level above which the BS configuration has to be modified. It measures the error tolerance of the network operator with respect to the random traffic variation. In a nutshell, our objective is to find the next time to update the BS configuration, in other words, solve the following problem:

$$
\begin{array}{ll}
\underset{\Delta t}{\operatorname{minimize}} & \Delta t \\
\text { subject to } & p\left(A_{1} \cup A_{2} \cup A_{3}\right) \geq \eta
\end{array}
$$

Solving this optimization problem analytically is a difficult task due to the intractability of the constraint. This is caused, for example, by complex expressions of the probability distribution functions of one of the events or their union. Instead, numerical methods such as Monte Carlo (MC) methods can be used. An iterative algorithm based on small-scale discretization parameter $\Delta t_{0}$ is proposed in Algorithm 1. The choice of low values of $\Delta t_{0}$ offers more accurate update decision making but leads to a higher execution time of the algorithm and vice versa. Note that the output $\Delta t$ of the above algorithm indicates that, at $t=t_{0}+\Delta t$, the considered BS configuration $\boldsymbol{\epsilon}, n \in\{1,2, \cdots, N\}$, has to be updated. In the following section, we present the probabilistic algorithm to update the BS configuration.

\section{B. Second Step: Update of the BS Configuration}

This section is devoted to determine the updated values of $\left\{\boldsymbol{\epsilon}_{n}\right\}_{n=1}^{N}$. It takes as input the time $t=t_{0}+\Delta t$ determined from Algorithm 1 and aims to update the BS configuration that has to be used in the next time period. As initial configuration, the proposed algorithm takes the BS configuration of the previous time period, i.e., the BS configuration between 


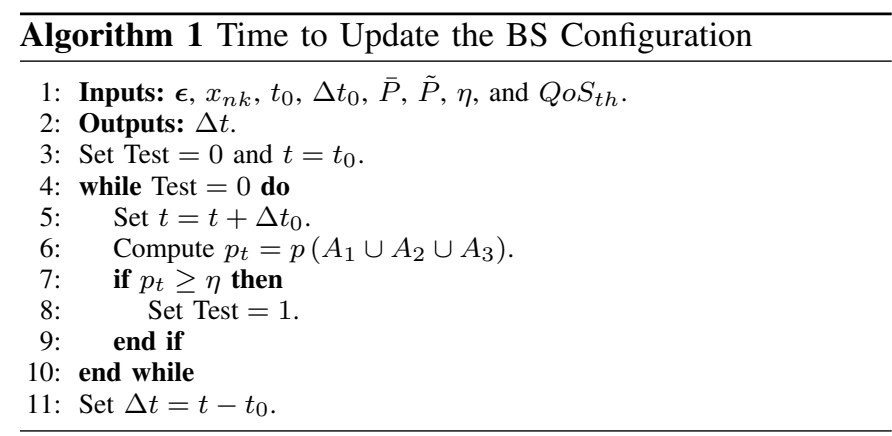

$\left[t_{0}, t_{0}+\Delta t\right)$ which denotes the interval between $t_{0}$ and $t_{0}+\Delta t$ which does not include $t_{0}+\Delta t$, and tries to update it by solving the following optimization problem:

$$
\begin{array}{ll}
\underset{\left\{\boldsymbol{\epsilon}_{n}\right\}_{n=1}^{N}}{\operatorname{minimize}} & \sum_{n=1}^{N} \boldsymbol{\epsilon}_{n} P_{n}\left(t_{0}+\Delta t, E\left[\tilde{N}_{n}\left(t_{0}+\Delta t\right)\right]\right) \\
\text { subject to } & p\left(A_{1} \cup A_{3}\right) \leq \eta^{\prime},
\end{array}
$$

where $E[\cdot]$ is the expected value operation. More precisely, we aim to activate BSs that minimize the expected value of the total consumed power at time instant $t_{0}+\Delta t$ under the constraint that the QoS and the BS power consumption constraints hold with a probability higher than $1-\eta^{\prime}$ with $\eta^{\prime} \leq \eta$. The choice of $\eta^{\prime}$, e.g., $\eta^{\prime}=\frac{\eta}{2}$, is made to ensure that the next time at which the BS configuration should be updated is not close to $t$ and hence, it contributes in minimizing the number of on/off oscillation rates. The above combinatorial optimization is difficult to solve analytically. However, we provide a low complex algorithm that yields an approximate solution. The proposed algorithm is divided into two sub-algorithms. First, if the constraint (11) is satisfied, we investigate whether we can switch off some of the BSs while satisfying (11). The subalgorithm corresponding to the previous situation is described in Algorithm 2a. The second sub-algorithm corresponds to the case where (11) is not satisfied (see Algorithm 2b). In this setting, the BS configuration is updated by switching on some of the BSs until the constraint (11) becomes satisfied. In both algorithms, one BS is turned off or on at each iteration. The selected BS is the one that, when turned off or on, provides the minimum power consumption. Hence, at each iteration, the algorithms test all the BSs by turning off or on one of them at each time. The probability in (11) and the objective function (10) are computed at each test to determine the BS to be activated or deactivated. Algorithm 2a converges when no more BS can be turned off while Algorithm $2 b$ converges when the constraint (11) becomes satisfied. Note also that Algorithm 2a and Algorithm 2b require $O\left(N^{2}\right)$ iterations to converge to their sub-optimal solutions. This leads to a low complexity compared to the one of the exhaustive search method whose complexity is of the order of $2^{N}$.

\section{Time-Varying and Dynamic ON/OFF Switching Algorithm}

In this section, we provide the iterative algorithm containing all steps of the proposed on/off switching approach for a period starting from $t=0$ to $t=T$. The final pseudo code is given in Algorithm 3. It should be noted that, after executing Algorithm $2 b$, it might be interesting to turn off additional BSs that were active before performing the update. Thus, the procedure in
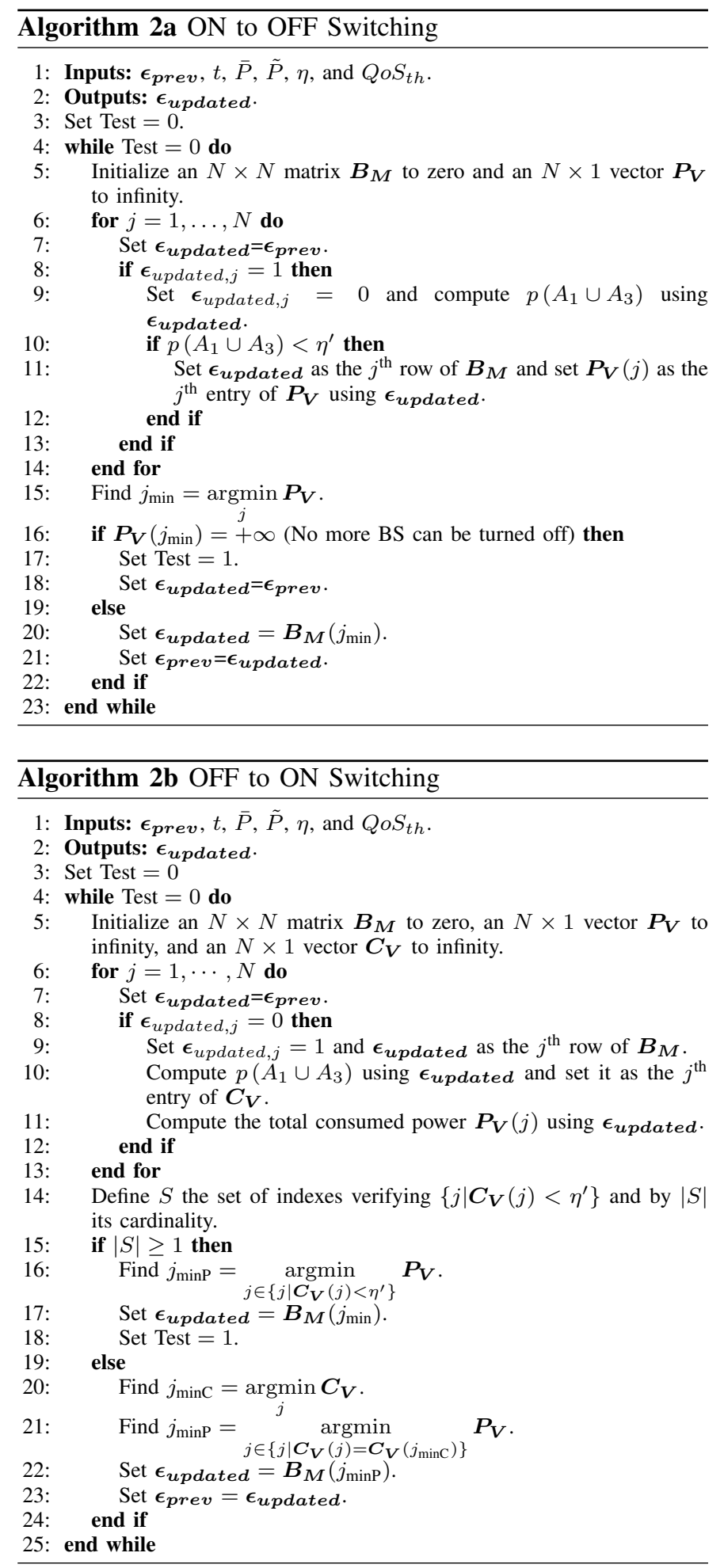

Algorithm 2a is called again to decide whether some active BSs can turned off while keeping (11) satisfied.

\section{Simulation Results And Concluding Remarks}

In this section, we apply the proposed on/off switching algorithm for a cellular network composed of $N=9$ BSs during an interval of time $[0, T]$ with $T=24$ hours. The BSs are placed in a uniform grid of size $3 \times 3 \mathrm{~km}^{2}$ with intersite distance equals to $1 \mathrm{~km}$. The parameters of the power consumption model are $a=4.7, P_{u}=0.8 \mathrm{~W}$, and $b=130$ $\mathrm{W}$ [6]. The number of users is modeled as follows: 


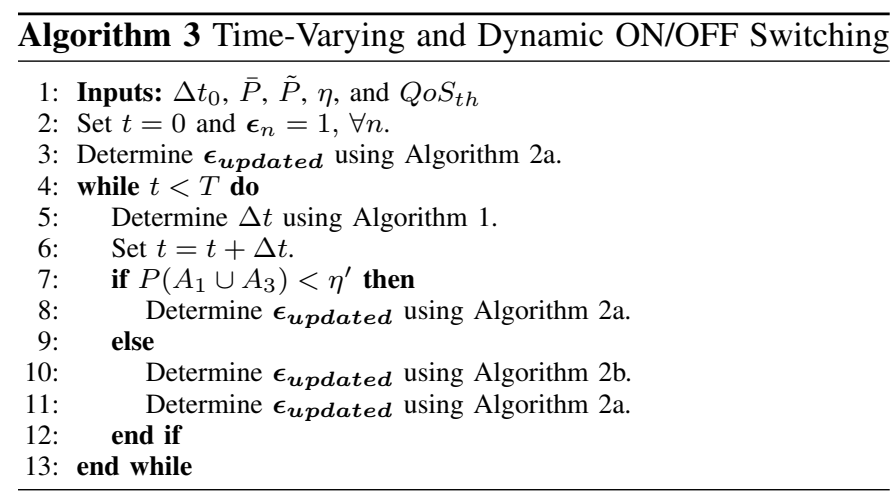

TABLE I: Time Intervals and BS Configurations

\begin{tabular}{|c|c|c|c|c|c|c|c|c|c|}
\hline & \multicolumn{10}{|c|}{ BS Configuration } \\
\hline Time interval & 1 & 2 & 3 & 4 & 5 & 6 & 7 & 8 & 9 \\
\hline \hline$[0,1.7)$ & 1 & 1 & 1 & 1 & 0 & 1 & 1 & 1 & 0 \\
\hline$[1.7,5.6)$ & 1 & 1 & 1 & 1 & 1 & 1 & 1 & 1 & 0 \\
\hline$[5.6,6.7)$ & 1 & 0 & 1 & 1 & 1 & 1 & 1 & 1 & 1 \\
\hline$[6.7,8.1)$ & 1 & 0 & 1 & 0 & 1 & 1 & 1 & 1 & 1 \\
\hline$[8.1,11.2)$ & 1 & 0 & 1 & 0 & 1 & 0 & 1 & 1 & 1 \\
\hline$[11.2,12.3)$ & 1 & 1 & 1 & 0 & 1 & 0 & 1 & 1 & 1 \\
\hline$[12.3,17.8)$ & 1 & 1 & 1 & 1 & 1 & 0 & 1 & 1 & 1 \\
\hline$[17.8,24]$ & 1 & 1 & 1 & 1 & 0 & 1 & 0 & 1 & 1 \\
\hline
\end{tabular}

$$
\bar{N}_{n}(t)=\left\{\begin{array}{l}
5+80 \phi\left(\frac{t-10}{2}\right)+60 \phi(t-16), \text { if } n=1,3,5,7,9, \\
5+40 \phi\left(\frac{t+4}{4}\right)+60 \phi\left(\frac{t-21}{6}\right), \text { if } n=2,4,6,8,
\end{array}\right.
$$

where $\phi(\cdot)$ is the standard normal probability density function. Moreover, the error terms $\delta_{n}(t)$ are assumed to be independent in $t$ and $n$ and follow a uniform distribution over the interval $[-\delta, \delta]$ with $\delta=2$. The parameters $Q o S_{t h}, \bar{P}$, and $\tilde{P}$ are selected to be equal to $0.9,4 a P_{u} N \mathrm{~W}$, and $50 a P_{u}+b \mathrm{~W}$, respectively. Finally, the time step $\Delta t_{0}$ is equal to 6 minutes whereas the risk levels $\eta$ and $\eta^{\prime}$ are $10 \%$ and $5 \%$. Table I presents the output of the proposed algorithm where the obtained time periods are given along with the corresponding BS configurations. In the period between 0 and 1.7 hours, BS 5 and 9 are deactivated. Then, at $t=1.7$ hours, the QoS constraint becomes unsatisfied with a probability higher than 0.1 . Therefore, the algorithm adapts the BS configuration by switching on BS 5 . The same observation occurs at $t=5.6$ hours since the number of users of BSs 1, 3, 5, 7 and 9 have increased. Thus, the BS statuses are updated by switching on BS 9. Also, at this time, the algorithm switches off BS 2 in order to reduce the power consumption without harming the system performances. In the period between 6.7 hours and 11.2 hours, the BS statuses are updated by first switching off BS 4 in the period between 6.7 hours and 8.1 hours and then, deactivating BS 6 from 8.1 hours to 11.2 hours. This is expected since the load in BSs $1,3,5,7$ and 9 has increased significantly compared to that of BSs $2,4,6$, and 8 . At time $t=11.2$ hours, the QoS constraint becomes unsatisfied within a risk higher than $10 \%$. Therefore, in order to retrieve a good network operation, BS 2 is activated in the period between 11.2 hours and 12.3 hours. As the loads of BSs 2, 4, 6 , and 8 continue to increase, the QoS criterion is again not satisfied at time $t=12.3$ hours. Thus, the algorithm switches off BS 4 and keeps this configuration until 17.8 hours. Finally, in the period between 17.8 hours and 24 hours, the algorithm switches on

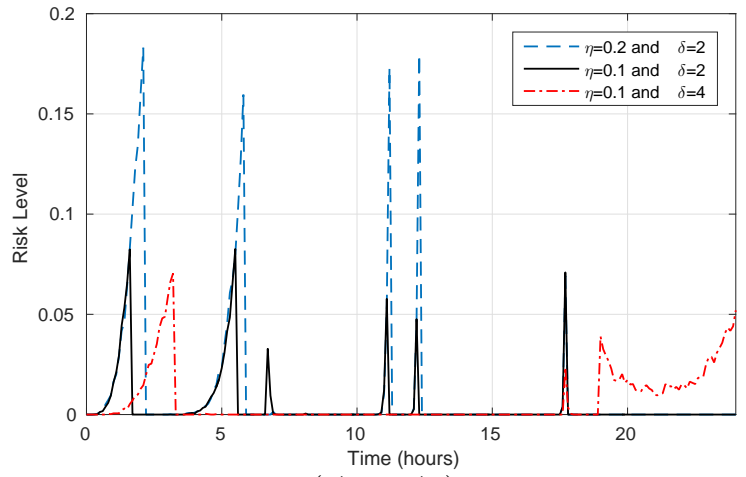

Fig. 1: Risk Level $p\left(A_{1} \cup A_{3}\right)$ as a function of time.

BS 6 and deactivates BSs 5 and 7. Such a result is expected since the number of users in this period has significantly decreased for BSs $1,3,5,7$, and 9 .

The next step is to investigate whether the on/off switching algorithm meets the risk level requirement for various values of system parameters. To this end, we plot in Fig. 1 the probability of risk $p\left(A_{1} \cup A_{3}\right)$, that is the probability that the QoS requirement or the power constraint does not hold, as a function of time and for different values of $\eta$ and $\delta$. From this figure and as expected, we observe that the probability of risk does not exceed $\eta$ for the whole period. Thus, we conclude that the proposed on/off switching meets the risk level requirement. Moreover, for $\eta=0.1$ and $\delta=2$, the peaks in Fig. 1 are in accordance with the results in Table 1. In fact, they correspond to the instants at which the proposed algorithm updates the BS configuration in order to meet the risk level requirement.

In a nutshell, we developed a time-varied probabilistic BSs on/off switching algorithm that adaptively updates the BSs configuration in order to reduce the average total power consumption. Moreover, we showed that this approach meets the risk requirement set by the operator. In addition to its economical and environmental benefits as discussed in the introduction, the proposed approach represents a practical solution for mobile operator to estimate the operations of their BSs during non-periodic time intervals allowing to better assess their traffic and energy management.

\section{REFERENCES}

[1] C. A. Chan, W. Li, S. Bian, I. Chih-Lin, A. F. Gygax, C. Leckie, M. Yan, and K. Hinton, "Assessing network energy consumption of mobile applications," IEEE Commun. Mag., vol. 53, no. 11, pp. 182 191, Nov. 2015.

[2] H. Ghazzai, E. Yaacoub, A. Kadri, H. Yanikomeroglu, and M. S. Alouini, "Next-generation environment-aware cellular networks: Modern green techniques and implementation challenges," IEEE Access, vol. 4, pp. 5010-5029, Sept. 2016.

[3] T. Han and N. Ansari, "A traffic load balancing framework for softwaredefined radio access networks powered by hybrid energy sources," IEEE/ACM Trans. Netw., vol. 24, no. 2, no. 2, pp. 1038-1051, Apr. 2016.

[4] J. Wu, Y. Bao, G. Miao, S. Zhou, and Z. Niu, "Base-station sleeping control and power matching for energy-delay tradeoffs with bursty traffic," IEEE Trans. Veh. Technol., vol. 65, no. 5, pp. 3657-3675, May. 2016.

[5] F. Han, S. Zhao, L. Zhang, and J. Wu, "Survey of strategies for switching off base stations in heterogeneous networks for greener 5G systems," IEEE Access, vol. 4, pp. 4959-4973, Aug. 2016.

[6] "Energy efficiency analysis of the reference systems, areas of improvements and target breakdown," Energy Aware Radio and neTwork tecHnologies, (EARTH), Dec. 2010.

[7] X. Guo, Z. Niu, S. Zhou, and P. R. Kumar, "Delay-constrained energyoptimal base station sleeping control," IEEE J. Sel. Areas Commun., vol. 34, no. 5, pp. 1073-1085, May 2016. 